\title{
Determinants of glucose control in patients with chronic pancreatitis
}

\author{
H. Schrader - B. A. Menge - C. Zeidler • P. R. Ritter • \\ A. Tannapfel • W. Uhl • W. E. Schmidt • J. J. Meier
}

Received: 17 September 2009 / Accepted: 3 February 2010 / Published online: 9 March 2010

(C) Springer-Verlag 2010

\begin{abstract}
Aims/hypothesis Diabetes frequently develops in patients with chronic pancreatitis (CP). Partial pancreatectomy has emerged as a treatment option for such patients. We addressed whether the development of diabetes in $\mathrm{CP}$ patients is related to pancreatic beta cell area or clinical variables, and which factors predict the diabetes risk after partial pancreatectomy.

Methods Fractional beta cell area was determined in pancreatic tissue samples obtained from $114 \mathrm{CP}$ patients undergoing pancreatic surgery and related to measures of glucose control, as well as clinical and anthropometric data. Seventy-four patients without diabetes at the time of surgery were contacted again $2.5 \pm 1.0$ years after partial pancreatectomy in order to obtain information about the post-operative development of diabetes.

Results In the surgical samples in the whole cohort, pancreatic beta cell area was $0.40 \pm 0.06 \%$ in patients with and $0.64 \pm 0.06 \%$ in those without previously known diabetes
\end{abstract}

H. Schrader and B. A. Menge contributed equally to this study.

H. Schrader • B. A. Menge • C. Zeidler • P. R. Ritter •

W. E. Schmidt $\cdot$ J. J. Meier $(\triangle)$

Department of Medicine I, St Josef-Hospital,

Ruhr-University Bochum,

Gudrunstr. 56,

44791 Bochum, Germany

e-mail: juris.meier@rub.de

W. Uhl

Department of Surgery, St Josef-Hospital,

Ruhr-University Bochum,

Bochum, Germany

A. Tannapfel

Department of Pathology, Ruhr-University Bochum,

Bochum, Germany $(p=0.039)$. There was an inverse non-linear relationship between pancreatic beta cell area and fasting glucose concentrations $(r=0.29)$ as well as $\mathrm{HbA}_{1 \mathrm{c}}$ levels $(r=0.36)$. Nineteen out of 74 previously normoglycaemic patients (26\%) developed diabetes over an average period of 2.5 years of follow-up. Pre-operative fasting glucose levels, $\mathrm{HbA}_{1 \mathrm{c}}$ and BMI were identified as predictors of diabetes after partial pancreatectomy. However, pancreatic beta cell area did not differ in those who subsequently developed diabetes $(0.66 \pm 0.15 \%)$ and those who did not $(0.62 \pm$ $0.08 \%, p=0.45$ ).

Conclusions/interpretation Hyperglycaemia in CP patients is associated with reduced beta cell area. However, reduced beta cell area does not predict the development of diabetes, suggesting that other factors are more important determinants of alterations in glucose metabolism in patients with $\mathrm{CP}$.

Keywords Beta cell area - Chronic pancreatitis - Diabetes · Glucose control · Inflammation · Pancreas .

Pancreatic surgery
Abbreviations
$\mathrm{CP} \quad$ Chronic pancreatitis
CT Computed tomography
IFG Impaired fasting glucose
NFG Normal fasting glucose

\section{Introduction}

The pathogenesis of diabetes involves a variable combination of reduced beta cell mass, beta cell dysfunction and insulin resistance [1-4]. In type 2 diabetes, hyperglycaemia has been associated with a deficit in beta cell mass of $\sim 20$ $65 \%$ of normal [5-11], and studies in rodent models of 
autoimmune diabetes or in patients with newly onset type 1 diabetes indicate that the extent of beta cell loss amounts to $\sim 50-70 \%$ around the manifestation of type 1 diabetes [12-14]. Diabetes is also frequently found in patients with chronic pancreatitis (CP), secondary to the inflammatory process within the pancreas [15-17]. Previous studies have shown that beta cell mass is also significantly reduced in patients with $\mathrm{CP}$, even though the islet destruction appears to occur relatively late during the clinical course of the disease [17]. However, conflicting data have been reported with regard to the prevalence of diabetes in patients with $\mathrm{CP}$ and its relationship to disease duration $[15,16,18]$.

In clinical practice, patients with diabetes secondary to $\mathrm{CP}$ are often characterised by the early need for exogenous insulin supplementation [16]. Whether the onset of hyperglycaemia and the quality of glucose control in these patients are directly related to the islet destruction within the pancreas is yet unclear. In addition, the impact of other risk factors, such as age and BMI, on the risk of developing diabetes in patients with $\mathrm{CP}$ has been less well studied.

Pancreatic surgery has emerged as a potential treatment option for patients with $\mathrm{CP}$ and severe intractable abdominal pain resistant to conservative analgetic treatment $[19,20]$. Indeed, the quality of life has been shown to improve significantly after partial pancreatectomy in patients with persistent abdominal pain due to CP [21]. However, as beta cell mass is also reduced substantially by a partial pancreatectomy, any such intervention bears the potential risk of promoting the development of diabetes [22, 23]. Therefore, it is important to identify clinical predictors of postoperative hyperglycaemia in order to allow for a better risk-benefit assessment before subjecting such patients to pancreatic surgery.

On that background, we addressed the following questions: (1) Is there a relationship between pancreatic beta cell area and glucose control in patients with CP? (2) What clinical characteristics are associated with the development of diabetes in patients with CP? (3) What are the predictors of postoperative diabetes development in patients with $\mathrm{CP}$ undergoing partial pancreatectomy?

\section{Methods}

Study design Pancreatic tissue was obtained from 114 patients undergoing pancreatic surgery for $\mathrm{CP}$ between February 2004 and September 2007. Fractional beta cell area was determined in all tissue samples, and various measures of glucose control and results of clinical laboratory tests as well as anthropometric data were collected from the patients' records by retrospective analysis. The study protocol was approved by the ethics committee of the
Ruhr-University Bochum (registration number 2528), and all patients gave informed consent before surgery.

Patients In total, 114 patients (74 men, 40 women) undergoing pancreatic resections in the Department of Surgery, St Josef-Hospital, Ruhr-University Bochum, between February 2004 and September 2007 were included. The clinical diagnosis of CP was confirmed by an independent pathologist in all cases. Proximal pancreaticoduodenectomies were performed in 94 patients, distal pancreatectomies were carried out in 15 patients and the entire pancreas was removed in five patients. Thus, in 98 patients the pancreatic head was examined, whereas in the remaining 16 patients the pancreatic tail was available. Pancreatic surgery was performed because of chronic abdominal pain resistant to analgetic medication, pancreatic duct obstruction or unclear pancreatic imaging results potentially suspicious for carcinoma. The clinical causes of $\mathrm{CP}$ included chronic alcohol consumption in 50 patients, structural pancreatic abnormalities (pancreas divisum) in 14 patients, autoimmune pancreatitis in three patients, benign pancreatic adenomas in four cases, obstructions of the common biliary duct or the papilla vateri in four patients and hereditary pancreatitis in two cases. One patient developed CP after an episode of acute biliary pancreatitis, and in 36 patients the aetiology of $\mathrm{CP}$ remained cryptic. One hundred and four patients were treated with pancreatic enzyme preparation, whereas ten patients did not receive any enzyme supplementation.

The presence of diabetes was determined by careful evaluation of the patient history as well as by the use of glucose-lowering medication. Thus, diabetes was previously known in 28 patients (treated with insulin in 15 cases, sulfonylureas/meglitinides in two cases, metformin in two cases, oral combination therapies in two cases and diet in seven cases), whereas the other patients had no history of known diabetes. The mean diabetes duration was $72.9 \pm$ 99.2 months.

Data collection All patient records were evaluated to collect anthropometric data and information about concomitant medical conditions, current drug medication and relevant laboratory results. Furthermore, all fasting glucose values recorded pre-operatively during the hospital stay were recorded. An average of $5.2 \pm 4.9$ fasting glucose values was recorded to calculate the mean fasting glucose concentrations. The diagnosis of diabetes was based on the patients' histories as well as on the pre-operative use of glucoselowering agents.

Prospective follow-up All patients undergoing pancreatic surgery were contacted again in October 2008 after a mean follow-up time of $2.5 \pm 1.0$ years (range, 1.2 and 4.2 years) after pancreatic surgery. By these means, information about 
the vital status, the postoperative manifestation of diabetes, the date of the initial diagnosis of $\mathrm{CP}$ and the duration of symptoms suggestive of $\mathrm{CP}$ were obtained.

Pancreatic tissue processing Pancreas was fixed in formaldehyde and embedded in paraffin for subsequent analysis as previously described [24]. Sequential $5 \mu \mathrm{m}$ sections were stained for insulin using a guinea pig anti-insulin antibody (\#A 0564; DAKO, Golstrup, Denmark; lot no. 00001500) at 1:400 dilution and an alkaline phosphatase/ RED detection system (DAKO Real Envision Detection System, \#K 5007 and \#K 5005; lot no. 00025382 and lot no. 00025812 , respectively).

Morphometric analysis For the determination of the fractional beta cell areas, the entire pancreatic sections stained for insulin were imaged using a Zeiss Axioplan microscope equipped with a motorised stage $\times 100$ magnification $(\times 10$ objective). A tile image of the tissue section was generated using the 'Mosaix' tool of the software Axiovision, version 4.5. The fractional areas of the pancreas stained positive for insulin were digitally quantified using a colour-based threshold using Zeiss Axiovision software as previously described [24]. One pancreatic tissue section per case was included in this analysis. In order to minimise the potential error induced by the heterogeneous distribution of the islets within the pancreas, the entire pancreatic tissue section available was included in this analyses. By these means, a mean $\left( \pm \mathrm{SD}\right.$ ) tissue area of $1.24 \pm 1.05 \mathrm{~cm}^{2}$ (range 0.21 $6.84 \mathrm{~cm}^{2}$ ) per patient was analysed.

In order to determine the intra- and inter-observer error of these determinations, 20 pancreatic sections were analysed twice by the same investigator as well as independently by two different investigators. These analyses yielded an intra-observer variability of $6.0 \%$, and an inter-observer variability of $8.5 \%$.

Calculations and statistical analysis Patient characteristics are reported as means $\pm \mathrm{SD}$; results are presented as means \pm SEM. All variables were compared by two-sided $t$ tests. A $p$ value $<0.05$ was taken to indicate significant differences. Correlation analyses were carried out using GraphPad Prism 4 in linear or non-linear regression functions.

\section{Results}

Pre-operative determinants of glucose control Diabetes was previously known in 28 patients, whereas 86 patients were free of diabetes before surgery by history. When fasting glucose concentrations were used as an additional diagnostic tool, 57 patients presented with normal fasting glucose (NFG); impaired fasting glucose (IFG) was found in 24 patients, and 33 patients were classified as having diabetes. Pancreatic beta cell area was $0.40 \pm 0.06 \%$ in patients with previously known diabetes and $0.64 \pm 0.06 \%$ in those patients in whom diabetes had not been diagnosed before hospital admission $(p=0.039)$ - and $0.72 \pm 0.08 \%$ in NFG patients, $0.48 \pm 0.06 \%$ in IFG patients and $0.39 \pm$ $0.05 \%$ in diabetic patients, when the fasting glucose concentrations were also considered ( $p=0.0084$; Fig. 1).

There was a non-linear inverse relationship between fasting glucose levels and pancreatic beta cell area $(r=0.29$; Fig. 2). In a similar fashion, pre-operative $\mathrm{HbA}_{1 \mathrm{c}}$ levels were inversely related to beta cell area $(r=0.36, p=0.020$; Fig. 2). These relationships were no longer detectable when the relationships between beta cell area and either fasting glucose or $\mathrm{HbA}_{1 \mathrm{c}}$ levels were examined individually in the groups of patients with and without diabetes. There was no significant association between BMI and either fasting glucose or $\mathrm{HbA}_{1 \mathrm{c}}$ levels (details not shown).

In order to identify which factors were associated with the presence of diabetes in CP patients, various variables were compared between the patients with and without previously known diabetes (Table 1). These analyses revealed significant differences only for age $(p=0.0033)$ and pancreatic beta cell area $(p=0.038)$. By contrast, body weight, $\mathrm{BMI}$ and the duration of $\mathrm{CP}$ were not associated with the diagnosis of diabetes in these patients.

Predictors of postoperative glucose control After the follow-up period of $2.5 \pm 1.0$ years, nine out of the 114 patients undergoing pancreatic surgery had died $(8 \%)$, six patients $(5 \%)$ were lost to follow-up, five patients $(4 \%)$ were excluded because total pancreatectomies had been performed, and in 20 of the remaining patients (18\%) diabetes had already been present before pancreatic surgery. Among the 74 patients eligible for the follow-up study, 19 (26\%) had developed diabetes during the postoperative
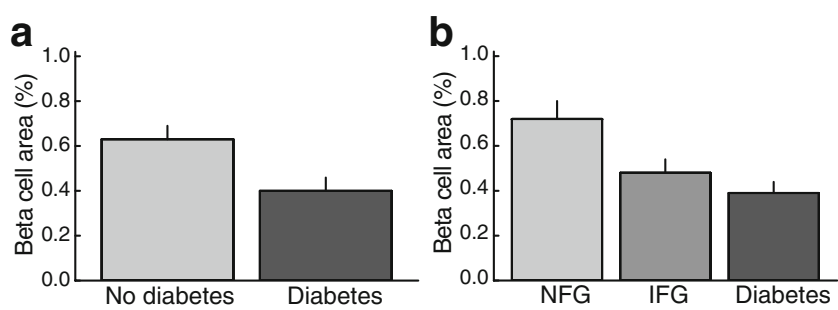

Fig. 1 Fractional beta cell area of the pancreas in 114 patients with chronic pancreatitis. Patients were grouped according to previous diagnosis of diabetes mellitus (a) or fasting glucose concentrations determined during the hospital stay (b). The $p$ values were 0.039 for (a) and 0.0084 for (b). Data are presented as means \pm SEM; statistics were carried out using the Student's $t$ test (a) or ANOVA (b), respectively 

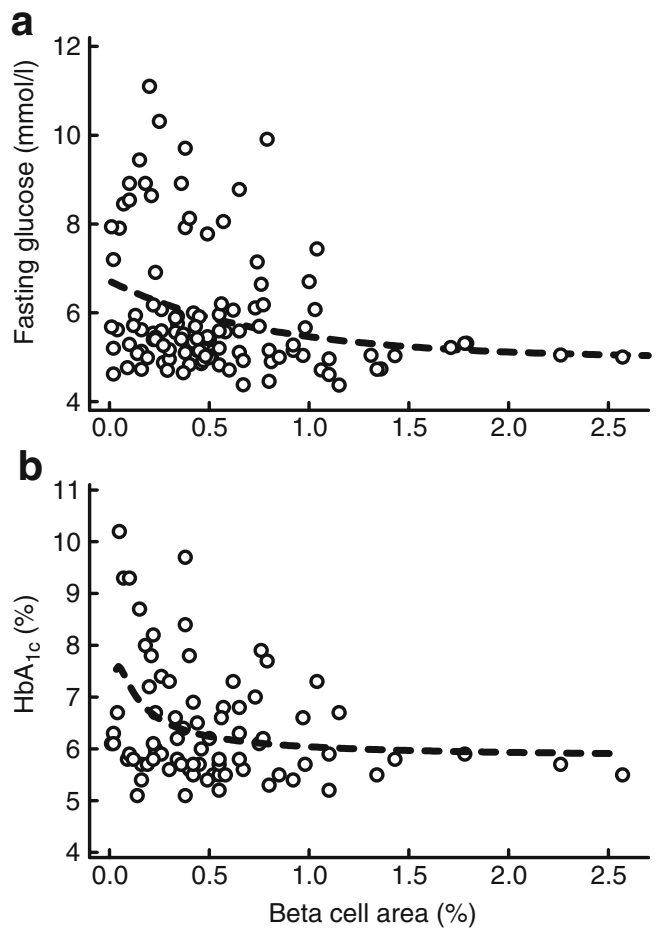

Fig. 2 Relationship between fasting glucose concentrations (a) or $\mathrm{HbA}_{1 \mathrm{c}}$ levels (b) and the fractional beta cell area of the pancreas in patients with chronic pancreatitis. Dashed lines indicate the regression lines calculated by non-linear regression analyses using a hyperbolic function. The calculated $r$ values were 0.29 and 0.36 for (a) and (b), respectively

follow-up period. In order to identify which factors determined the development of diabetes after partial pancreatectomy, all eligible patients without diabetes before surgery were divided into those developing diabetes during the postoperative follow-up period and those remaining free of diabetes. Based on these analyses, the pre-operative fasting glucose and $\mathrm{HbA}_{1 \mathrm{c}}$ levels, as well as the preoperative BMI, were identified as predictors of diabetes after surgery (Table 2). However, there was no difference in fractional beta cell area between the patients who did or did not develop diabetes after the partial pancreatectomy (Fig. 3). At the time of partial pancreatectomy beta cell area was $0.66 \pm 0.15 \%$ in those who subsequently developed diabetes, whereas in those who did not do so it was $0.62 \pm$ $0.08 \%(p=0.45)$. There also was no significant effect of the respective surgical procedure (pancreatic head vs tail resection) on the postoperative risk of diabetes (Table 2).

\section{Discussion}

The present studies were designed to examine the association between pancreatic beta cell area and various clinical characteristics and the development of diabetes in patients with $\mathrm{CP}$, and to identify potential predictors of postoperative diabetes development in patients undergoing partial pancreatectomies for the treatment of CP. Based on the analysis of histological and clinical data from 114 patients with histologically confirmed $\mathrm{CP}$, we report that: (1) pancreatic beta cell area is significantly lower in CP patients with diabetes compared with non-diabetic patients; (2) there is an inverse non-linear relationship between pancreatic beta cell area and fasting glucose or $\mathrm{HbA}_{1 \mathrm{c}}$ levels; and (3) obesity and pre-operative fasting glycaemia determine the individual risk of diabetes development after partial pancreatectomy.
Table 1 Characteristics of 114 patients with $\mathrm{CP}$ with and without known diabetes before pancreatic surgery

Data are presented as means \pm SEM

Numbers in parentheses indicate the total number of patients in whom the respective information was available

${ }^{a}$ Fisher's exact test or Student's $t$ test

\begin{tabular}{llll}
\hline Variable & With diabetes & Without diabetes & $p$ value $^{a^{a}}$ \\
\hline Age (years) & $57.2 \pm 2.1(28)$ & $48.8 \pm 1.4(86)$ & 0.0033 \\
Sex (\% male/female) & $79 / 21(28)$ & $60 / 40(86)$ & 0.11 \\
Body weight (kg) & $68.9 \pm 2.0(28)$ & $68.6 \pm 1.54(84)$ & 0.88 \\
Body height (m) & $1.75 \pm 0.01(28)$ & $1.74 \pm 0.01(84)$ & 0.76 \\
BMI (kg/m $\left.{ }^{2}\right)$ & $22.7 \pm 0.7(28)$ & $22.6 \pm 0.4(84)$ & 0.89 \\
Duration of symptoms (months) & $71.8 \pm 12.6(25)$ & $69.8 \pm 9.1(73)$ & 0.50 \\
Time since diagnosis (months) & $69.5 \pm 18(24)$ & $50.1 \pm 7.8(73)$ & 0.28 \\
Fasting glucose (mmol/l) & $7.41 \pm 0.34(28)$ & $5.49 \pm 0.09(85)$ & $<0.0001$ \\
HbA 1 (\%) & $7.48 \pm 0.24(26)$ & $5.88 \pm 0.08(52)$ & $<0.0001$ \\
Amylase (U/l) & $92.5 \pm 38.9(28)$ & $196.0 \pm 61.3(85)$ & 0.35 \\
Lipase (U/l) & $34.7 \pm 9.9(18)$ & $73.8 \pm 22.0(57)$ & 0.31 \\
Carcinoembryonic antigen (U/l) & $4.2 \pm 1.5(26)$ & $2.9 \pm 0.2(76)$ & 0.24 \\
Carbohydrate antigen 19-9 (U/l) & $41.7 \pm 18.2(28)$ & $24.5 \pm 4.4(76)$ & 0.10 \\
Aspartate aminotransferase (U/l) & $34.4 \pm 5.6(28)$ & $37.6 \pm 8.5(86)$ & 0.82 \\
Gamma-glutamyltransferase (U/l) & $134.5 \pm 35.2(28)$ & $133.1 \pm 30.4(86)$ & 0.99 \\
Alcohol abuse (\% current/never/past) & $29 / 39 / 32(28)$ & $23 / 49 / 27(79)$ & 0.62 \\
Nicotine abuse (\% yes/no/past) & $68 / 25 / 4(28)$ & $66 / 29 / 5(80)$ & 0.91 \\
\hline
\end{tabular}


Table 2 Characteristics of 74 patients with CP who did or did not develop diabetes after partial pancreatectomy

Data are presented as means \pm SEM

Numbers in parentheses indicate the total number of patients in whom the respective information was available

${ }^{a}$ Fisher's exact test or Student's $t$ test

\begin{tabular}{llll}
\hline Variable & With diabetes & Without diabetes & $p$ value $^{\mathrm{a}}$ \\
\hline Age (years) & $53.8 \pm 2.3(19)$ & $47.3 \pm 1.8(55)$ & 0.0534 \\
Sex (\% male/female) & $53 / 47(19)$ & $65 / 35(55)$ & 0.18 \\
Body weight (kg) & $71.4 \pm 3.3(18)$ & $67.0 \pm 14.1(55)$ & 0.25 \\
Body height (m) & $1.72 \pm 0.02(18)$ & $1.74 \pm 0.01(55)$ & 0.31 \\
BMI (kg/m $\left.{ }^{2}\right)$ & $24.1 \pm 0.9(18)$ & $21.9 \pm 0.5(55)$ & 0.030 \\
Duration of symptoms (months) & $64.6 \pm 19.7(18)$ & $63.6 \pm 8.0(51)$ & 0.96 \\
Time since diagnosis (months) & $43.7 \pm 15.5(18)$ & $45.2 \pm 7.8(51)$ & 0.93 \\
Fasting glucose (mmol/l) & $6.11 \pm 0.26(19)$ & $5.24 \pm 0.09(54)$ & 0.0002 \\
HbA 1 (\%) & $6.22 \pm 0.18(14)$ & $5.75 \pm 0.08(31)$ & 0.0096 \\
Amylase (U/l) & $48.4 \pm 14.1(18)$ & $90.9 \pm 34.3(55)$ & 0.49 \\
Lipase (U/l) & $56.2 \pm 14.9(12)$ & $260.1 \pm 91.3(38)$ & 0.22 \\
Carcinoembryonic antigen (U/l) & $3.8 \pm 0.8(16)$ & $2.7 \pm 0.2(49)$ & 0.082 \\
Carbohydrate antigen 19-9 (U/l) & $20.7 \pm 6.1(16)$ & $20.7 \pm 5.4(49)$ & 0.998 \\
Aspartate aminotransferase (U/l) & $30.2 \pm 6.1(19)$ & $28.5 \pm 3.5(55)$ & 0.81 \\
Gamma-glutamyltransferase (U/l) & $63.4 \pm 14.7(19)$ & $168.9 \pm 47.6(55)$ & 0.20 \\
Alcohol abuse (\% current/never/past) & $18 / 47 / 35(17)$ & $20 / 49 / 29(51)$ & 0.92 \\
Nicotine abuse (\% yes/no/past) & $59 / 35 / 6(17)$ & $71 / 23 / 6(52)$ & 0.60 \\
Pancreatic resection (\% head/tail) & $79 / 21(19)$ & $85 / 15(55)$ & 0.49 \\
\hline
\end{tabular}

An inverse relationship between pancreatic beta cell area and fasting glucose concentrations has previously been reported in patients with type 2 diabetes and non-diabetic patients based on the analysis of autopsy tissue [9]. The present study extends these findings by demonstrating a similar relationship in patients with diabetes secondary to $\mathrm{CP}$ and by providing an association between beta cell area and $\mathrm{HbA}_{1 \mathrm{c}}$ levels for the first time.

Nevertheless, the characteristics of type 2 diabetes and diabetes secondary to $\mathrm{CP}$ seem to differ substantially from each other: thus, the individual risk for type 2 diabetes is clearly increased in obese individuals [25], most likely as a result of impaired insulin action. By contrast, BMI was not associated with either fasting glucose or $\mathrm{HbA}_{1 \mathrm{c}}$ levels in the present group of patients with CP. Also, the mean BMI of these patients $\left(\sim 23 \mathrm{~kg} / \mathrm{m}^{2}\right)$ was far lower than typically found in patients with type 2 diabetes [26]. However, there was a significant difference in BMI between patients developing diabetes after a partial pancreatectomy and those remaining normoglycaemic, even though the mean BMI was still within normal limits in both groups. This suggests that even small differences in body weight can increase the individual risk of diabetes after a partial pancreatectomy. Therefore, it is important to emphasise that the presently reported findings apply to patients with diabetes secondary to $\mathrm{CP}$ only and should not be generalised to the group of patients with type 2 diabetes.

The significant association between pancreatic beta cell area and $\mathrm{HbA}_{1 \mathrm{c}}$ levels also suggests that the extent of beta cells in the pancreas is one important determinant of glycaemic control in diabetic patients. This is consistent with previous studies in patients with type 1 diabetes showing a clear relationship between beta cell function and $\mathrm{HbA}_{1 \mathrm{c}}$ levels during glucose-lowering treatment [27, 28]. The mechanisms underling this association have not been completely clarified yet, but may involve the effects of endogenous insulin on alpha cell function [29-31] as well as the direct intra-portal drainage of endogenous insulin vs systemic delivery of exogenously administered insulin [32]. Taken together, these studies emphasise the importance of endogenous insulin secretion for glucose control and support the concept of enhancing beta cell function in the treatment of patients with diabetes [33]. However, because the associations between beta cell area and glycaemic control described herein were purely correlative in nature, they do not allow for firm conclusions regarding the causality of this relationship. Thus, it also possible, that the presence of diabetes had a detrimental impact on beta

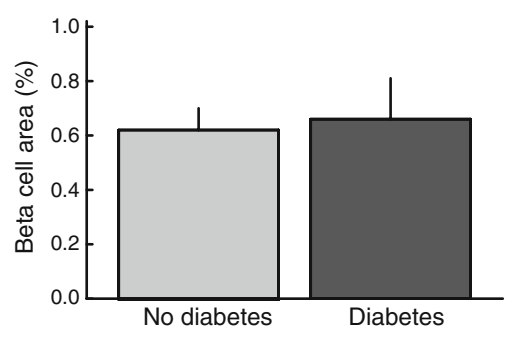

Fig. 3 Fractional beta cell area of the pancreas in 74 patients with chronic pancreatitis who did $(n=19)$ or did not $(n=55)$ develop diabetes within $2.5 \pm 1.0$ years after partial pancreatectomy. Data are presented as means \pm SEM. The $p$ value (Student's $t$ test) was 0.45 
cell area, possibly mediated through the mechanism of glucose toxicity. Consistent with such reasoning, the inverse relationship between beta cell area and glycaemic control was no longer detectable when the groups of patients with and without diabetes were studied separately.

The present results are in support of our previous study about functional predictors of beta cell area in humans [34]. In that study, an even closer association between glycaemia and beta cell area has been found. It is therefore important to bear in mind the differences between these studies. Thus, in the previous experiments oral glucose tolerance tests were carried out under controlled conditions and after prior withdrawal of all glucose-lowering drugs in a group of 33 patients with different pancreatic diseases. By contrast, the present data were based on the fasting glucose levels determined under normal conditions and without prior drug-washout in a large group exclusively comprised of patients with CP. This was done for practicability reasons and in order not to extend the pre-operative time of hospitalisation in these patients. Because of the frequent use of glucose-lowering medication in the patients with diabetes studied herein, it is likely that glucose concentrations of these patients would have been even higher after a drug-washout period. This might explain why the associations between beta cell area and glycaemic control found in this study were weaker than those described in the previous study [34].

Although this study has demonstrated significant differences in beta cell area between patients with and without diabetes, these differences could account for only a small percentage of the overall variations in glycaemia, and there was still a large degree of inter-individual variability in beta cell area within patients exhibiting similar fasting glucose concentrations. This is in agreement with previous studies in the pancreas of patients with and without type 2 diabetes $[9,11]$, and may on the one hand reflect the limitations associated with the analysis of a single piece of pancreatic tissue, the variability of the morphometric methods applied and the typical fluctuations of fasting glycaemia. On the other hand, the inter-individual heterogeneity in beta cell area could also suggest that glucose control in patients with $\mathrm{CP}$ is not only determined by pancreatic beta cell area, but also involves other factors, such as impairments in beta cell function and insulin resistance. Consistent with this, previous studies have demonstrated an impairment of the insulin secretory responses to various stimuli in $\mathrm{CP}$ patients, the extent of which seems to clearly exceed the beta cell deficit found in such patients $[18,35]$.

Some limitations may apply with respect to this study. In fact, even though formally the determination of beta cell mass requires both pancreatic weight and beta cell area [36], only beta cell area was measured in this study. This was done by necessity, because pancreatic weight cannot be determined in living humans. We have tried to overcome this problem previously by generating a measure of pancreatic volume based on abdominal computed tomography $(\mathrm{CT})$ scans in a controlled subset of patients with $\mathrm{CP}$ without any other detectable pancreatic abnormalities [17]. In the present study, the clinical evaluation of the abdominal CT scans had revealed a pancreatic oedema in a considerable group of patients, thereby likely inducing bias with respect to the determination of pancreatic volume. Therefore, the present analyses have been based on the quantification of pancreatic beta cell area only. However, as the variations in pancreatic volume have previously been shown to be relatively small in adult humans [37], the overall impact of this factor would be expected to be rather minor. Furthermore, beta cell area can vary extensively within the pancreas, and this factor might be of particular relevance in patients with $\mathrm{CP}$, in whom different parts of the organ might be unequally affected by the inflammatory process. In this regard, some studies have also reported a preferential abundance of beta cells in the pancreatic tail [11], but this has not been confirmed by others [7, 34]. Nevertheless, the error implied by these factors might have contributed to the great variance in beta cell area observed in this study. In this regard, it should also be noted that more than $90 \%$ of the patients studied herein exhibited severe exocrine insufficiency requiring exogenous enzyme supplementation. Because exocrine insufficiency leading to maldigestion has also been shown to have an important impact on glucose control [38], this factor might have added to the variability in glycaemia in these patients.

The results of this study have important clinical implications for the assessment of the individual diabetes risk in patients with $\mathrm{CP}$ before a partial pancreatectomy. Thus, even though the overall percentage of patients developing diabetes after the intervention was relatively low (26\%), higher pre-operative BMI and fasting glucose levels were significantly associated with the postoperative manifestation of diabetes. As diabetes is associated with an increased morbidity and mortality and may independently worsen the quality of life, these potential risk factors should be borne in mind when subjecting patients with $\mathrm{CP}$ to pancreatic surgery. Nevertheless, these data also demonstrate that a large percentage of patients undergoing pancreatic surgery for CP remain free of diabetes, at least over a follow-up period of $\sim 2.5$ years.

In conclusion, the present study has established an inverse relationship between pancreatic beta cell area and glycaemic control in patients with CP. However, the large degree of inter-individual variability in beta cell area also suggests that other variables, such as beta cell function and insulin resistance, are also likely to be important for glucose homoeostasis in such patients. Furthermore, mild elevations in glycaemia and BMI have been identified as risk factors 
for the development of diabetes after partial pancreatectomy. This suggests that metabolic variables should be included in the individual risk-benefit assessment of pancreatic surgeries in patients with $\mathrm{CP}$.

Acknowledgements The excellent technical assistance of B. Baller, M. Schweinsberg, H. Achner and G. Müller is gratefully acknowledged. These studies were supported by grants from the Deutsche Forschungsgemeinschaft (DFG grant no. Me2096/5-1 to JJM) and the Ruhr-University of Bochum (FoRUM grants to JJM).

Duality of interest The authors declare that there is no duality of interest associated with this manuscript.

\section{References}

1. DeFronzo RA (1988) Lilly lecture 1987. The triumvirate: betacell, muscle, liver. A collusion responsible for NIDDM. Diabetes 37:667-687

2. Ward WK, Beard JC, Halter JB, Pfeifer MA, Porte D Jr (1984) Pathophysiology of insulin secretion in non-insulin-dependent diabetes mellitus. Diabetes Care 7:491-502

3. Reaven GM (1988) Banting Lecture: role of insulin resistance in human disease. Diabetes 37:1593-1607

4. Meier JJ, Butler PC (2005) Insulin secretion. In: DeGroot LJ, Jameson JL (eds) Endocrinology. Elsevier Saunders, Philadelphia, pp 961-973

5. Clark A, Wells CA, Buley ID et al (1988) Islet amyloid, increased A-cells, reduced $\mathrm{B}$ cells and exocrine fibrosis: quantitative changes in the pancreas in type 2 diabetes. Diabetes Res 9:151159

6. Kloppel G, Lohr M, Habich K, Oberholzer M, Heitz PU (1985) Islet pathology and the pathogenesis of type 1 and type 2 diabetes mellitus revisited. Surv Synth Pathol Res 4:110-125

7. Yoon KH, Ko SH, Cho JH et al (2003) Selective beta-cell loss and alpha-cell expansion in patients with type 2 diabetes mellitus in Korea. J Clin Endocrinol Metab 88:2300-2308

8. Butler AE, Janson J, Bonner-Weir S, Ritzel R, Rizza RA, Butler PC (2003) Beta-cell deficit and increased beta-cell apoptosis in humans with type 2 diabetes. Diabetes 52:102-110

9. Ritzel RA, Butler AE, Rizza RA, Veldhuis JD, Butler PC (2006) Relationship between beta-cell mass and fasting blood glucose concentration in humans. Diabetes Care 29:717-718

10. Rahier J, Goebbels RM, Henquin JC (1983) Cellular composition of the human diabetic pancreas. Diabetologia 24:366-371

11. Rahier J, Guiot Y, Goebbels RM, Sempoux C, Henquin JC (2008) Pancreatic beta-cell mass in European subjects with type 2 diabetes. Diabetes Obes Metab 10:32-42

12. Lohr M, Kloppel G (1987) Residual insulin positivity and pancreatic atrophy in relation to duration of chronic type 1 (insulin-dependent) diabetes mellitus and microangiopathy. Diabetologia 30:757-762

13. Meier JJ, Lin JC, Butler AE, Galasso R, Martinez DS, Butler PC (2006) Direct evidence of attempted beta-cell regeneration in an 89 -year old patient with recent onset type 1 diabetes. Diabetologia 49:1838-1844

14. Sreenan S, Pick AJ, Levisetti M, Baldwin AC, Pugh W, Polonsky KS (1999) Increased beta-cell proliferation and reduced mass before diabetes onset in the nonobese diabetic mouse. Diabetes 48:989-996

15. Lankisch PG (2001) Natural course of chronic pancreatitis. Pancreatology 1:3-14
16. Larsen S (1993) Diabetes mellitus secondary to chronic pancreatitis. Dan Med Bull 40:153-162

17. Schrader H, Menge BA, Schneider S et al (2009) Reduced pancreatic volume and beta-cell area in patients with chronic pancreatitis. Gastroenterology 136:513-522

18. Kalk WJ, Vinik AI, Jackson WP, Bank S (1979) Insulin secretion and pancreatic exocrine function in patients with chronic pancreatitis. Diabetologia 16:355-358

19. Jimenez RE, Fernandez-Del Castillo C, Rattner DW, Warshaw AL (2003) Pylorus-preserving pancreaticoduodenectomy in the treatment of chronic pancreatitis. World J Surg 27: $1211-1216$

20. Uhl W, Gloor B, Buchler MW (1999) Pancreatic surgery. Curr Opin Gastroenterol 15:410-419

21. Diener MK, Rahbari NN, Fischer L, Antes G, Buchler MW, Seiler CM (2008) Duodenum-preserving pancreatic head resection vs pancreatoduodenectomy for surgical treatment of chronic pancreatitis: a systematic review and meta-analysis. Ann Surg 247:950-961

22. Litwin J, Dobrowolski S, Orlowska-Kunikowska E, Sledzinski Z (2008) Changes in glucose metabolism after Kausch-Whipple pancreatectomy in pancreatic cancer and chronic pancreatitis patients. Pancreas 36:26-30

23. Menge BA, Schrader H, Breuer TG et al (2009) Metabolic consequences of a $50 \%$ partial pancreatectomy in humans. Diabetologia 52:306-317

24. Menge BA, Tannapfel A, Belyaev $O$ et al (2008) Partial pancreatectomy in adult humans does not provoke beta-cell regeneration. Diabetes 57:142-149 (Epub 2007 Oct 2024)

25. Rana JS, Li TY, Manson JE, Hu FB (2007) Adiposity compared with physical inactivity and risk of type 2 diabetes in women. Diabetes Care 30:53-58

26. UK Prospective Diabetes Study (UKPDS) Group (1998) Intensive blood-glucose control with sulphonylureas or insulin compared with conventional treatment and risk of complications in patients with type 2 diabetes (UKPDS 33). Lancet 352:837853

27. Madsbad S (1983) Prevalence of residual B cell function and its metabolic consequences in type 1 (insulin-dependent) diabetes. Diabetologia 24:141-147

28. Steffes MW, Sibley S, Jackson M, Thomas W (2003) Beta-cell function and the development of diabetes-related complications in the diabetes control and complications trial. Diabetes Care 26:832-836

29. Zhou H, Tran PO, Yang S et al (2004) Regulation of alpha-cell function by the beta-cell during hypoglycemia in Wistar rats: the "switch-off" hypothesis. Diabetes 53:1482-1487

30. Meier JJ, Kjems LL, Veldhuis JD, Lefèbvre P, Butler PC (2006) Post prandial suppression of glucagon secretion depends on intact pulsatile insulin secretion: further evidence for the intraislet insulin hypothesis. Diabetes 55:1051-1056

31. Raju B, Cryer PE (2005) Loss of the decrement in intraislet insulin plausibly explains loss of the glucagon response to hypoglycemia in insulin-deficient diabetes: documentation of the intraislet insulin hypothesis in humans. Diabetes 54:757764

32. Frystyk J, Ritzel RA, Maubach J et al (2008) Comparison of pancreas-transplanted type 1 diabetic patients with portal-venous vs systemic-venous graft drainage: impact on glucose regulatory hormones and the growth hormone/insulin-like growth factor-I axis. J Clin Endocrinol Metab 93:1758-1766

33. Meier JJ (2008) Beta cell mass in diabetes: a realistic therapeutic target? Diabetologia 57:703-713

34. Meier JJ, Menge BA, Breuer TGK et al (2009) Functional assessment of beta-cell mass in humans. Diabetes 58:15951603 
35. Larsen S, Hilsted J, Tronier B, Worning H (1987) Metabolic control and $\mathrm{B}$ cell function in patients with insulin-dependent diabetes mellitus secondary to chronic pancreatitis. Metabolism 36:964-967

36. Bonner-Weir S (2001) Beta-cell turnover: its assessment and implications. Diabetes 50(Suppl 1):S20-S24
37. Saisho Y, Butler AE, Meier JJ et al (2007) Pancreas volumes in humans from birth to age one hundred taking into account sex, obesity and presence of type 2 diabetes. Clin Anat 20:933-942

38. Ebert R, Creutzfeldt W (1980) Reversal of impaired GIP and insulin secretion in patients with pancreatogenic steatorrhea following enzyme substitution. Diabetologia 19:198-204 